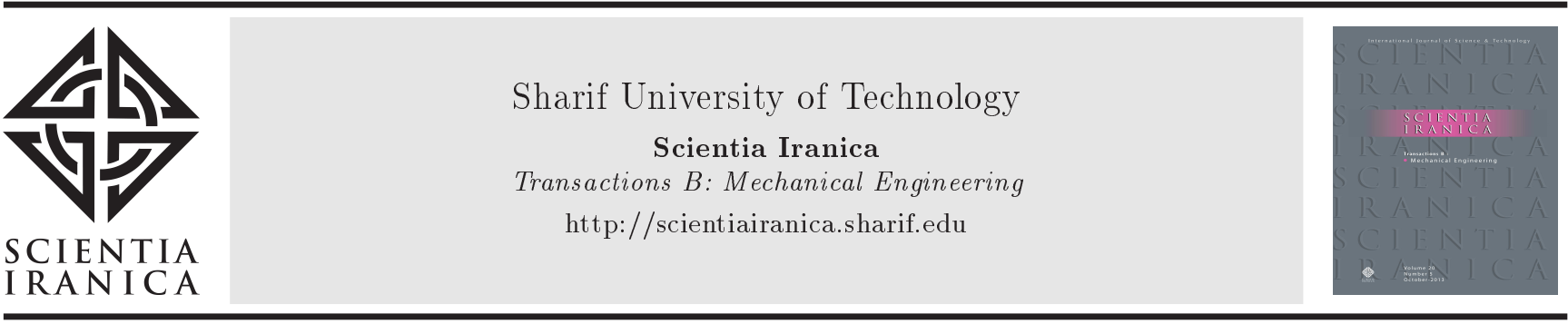

\title{
Hemodynamic analysis of coronary artery bypass grafting with elastic walls and different stenoses
}

\author{
H. Fallahi ${ }^{a, *}$, E. Shirani ${ }^{\mathrm{a}}$, and E. Zohravi ${ }^{\mathrm{b}}$ \\ a. Foulad Institute of Technology, Fouladshahr, Isfahan, P.O. Box 8491663763, Iran. \\ b. Department of Mechanical Engineering, Isfahan University of Technology, Isfahan, P.O. Box 8415683111, Iran. \\ Received 5 May 2019; received in revised form 31 October 2019; accepted 18 February 2020
}

KEYWORDS
Hemodynamics;
Bypass;
Atherosclerosis;
Coronary artery;
Fluid-solid
interaction;
Pulsatile blood flow.

\section{Introduction}

Many researchers have, thus far, investigated artery stenosis as the result of atherosclerosis [1]. Atherosclerosis means arterial stiffness due to the growth of blood platelets. In general, arteries must have flat and soft walls for easy transfer of red blood cells, oxygen, white blood cells, and nutrients so that vital materials can be provided for the body. The growth of blood platelets makes artery walls rigid and ultimately, causes vessels to narrow. The exact cause of atherosclerosis is unknown; however, it can be concluded that there has been a direct relationship between diet and the disease so far. A certain amount of cholesterol leads to the

\footnotetext{
*. Corresponding author.

E-mail address: human.fallahir@gmail.com (H. Fallahi)
}

doi: $10.24200 /$ sci. 2020.53378 .3211 enlargement of blood platelets. In general, atherosclerotic lesions in the coronary arteries are related to low and oscillating Wall Shear Stresses (WSS) [2]. Once the magnitude of the WSS exceeds 400 dynes $/ \mathrm{cm}^{2}$, the endothelium surface is irreversibly damaged [3]. Moreover, a number of studies have suggested that much Intimal Thickening (IT) occurs when the mean WSS is less than about 10 dynes $/ \mathrm{cm}^{2}[4,5]$.

This disease in the important arteries of the body such as coronary, carotid, femoral, and abdominal causes disturbance in blood to downstream regions and, finally, it is possible to cause lesion in downstream areas due to anemia $[6,7]$.

Based on a review of previous studies on the arterial stenosis, researchers have investigated stenosis quite well [8-12], but none of them have simulated the complete bypass graft considering elasticity of the vessel wall and pulsatility of flow simultaneously. Blood flow is assumed Newtonian here, as has been confirmed 


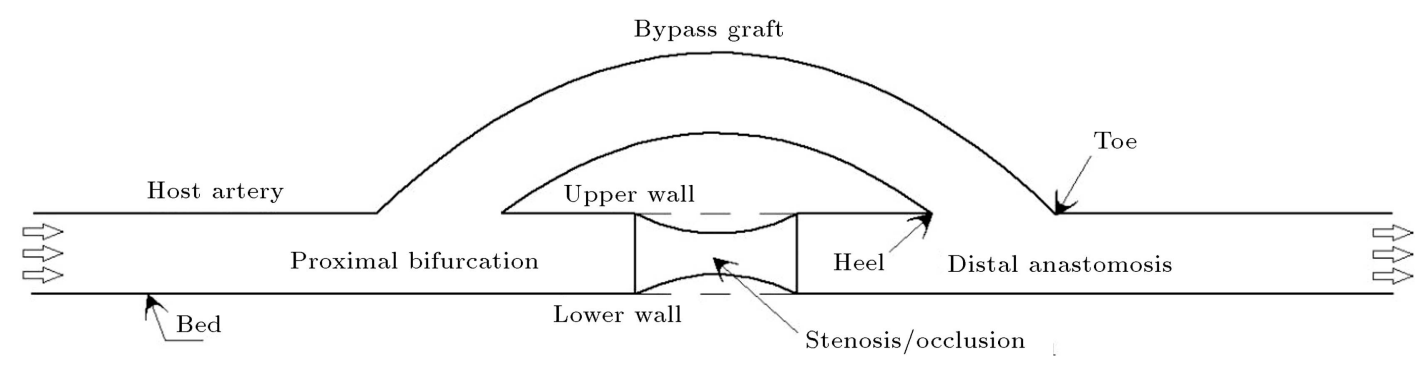

Figure 1. A full-frontal view of the bypass.

by many researchers [13-15]. This study considers the impacts of wall elasticity on fluid flow.

Hemodynamics play a critical role in the development and progression of plaques that are prone to sites of arterial curvatures and branch bifurcations. The occurrence of atherosclerosis at curvatures of coronary arteries and at the bifurcation sites of their branches is of medical importance $[16,17]$.

In 1996, assuming the Newtonian nature of blood, $\mathrm{He}$ and $\mathrm{Ku}$ [18] examined blood flow in the bifurcation area of coronary artery. They concluded that high spatial fluctuations in the values of shear stress and the low value of shear stress exerted on the vessel walls were heavily associated with the onset of atherosclerosis disease and the stenosis of arteries. Other studies conducted in this field include Rindt and Steenhoven [19], Weston et al. [20], Loudon and Tordesillas [21], Wentzel et al. [22], and Leuprecht et al. [23].

In this study, the elasticity of vessel wall is considered and blood flow is pulsatile. Another difference between this study and previous ones [2426] lies in considering complete bypass. This means that both distal and proximal anastomoses are modeled. Moreover, three different percentages of stenosis $(30 \%, 50 \%$, and $70 \%)$ are considered to determine the differences between the hemodynamic parameters of blood that might result in further stenosis by changing the stenosis percentages. Figure 1 shows the graft and complete bypass of the geometric model schematically.

\section{Materials and methods}

\subsection{Fluid-Solid Interaction (FSI) coupling}

According to the terms of the issue and its complexity, selecting the right approach to simulation can be different. In fact, this approach depends on the extent to which the fluid flow and solid body are affected by each other. According to this view, FSI problem can be divided into two types (Figure 2) [27]. First, the fluid flow field and solid body are highly interconnected, physically. In this case, the problem is too difficult to solve and the coupling between two fields is completely two-way. Second, the fluid flow and the solid body are almost independent of each other. In this case, there

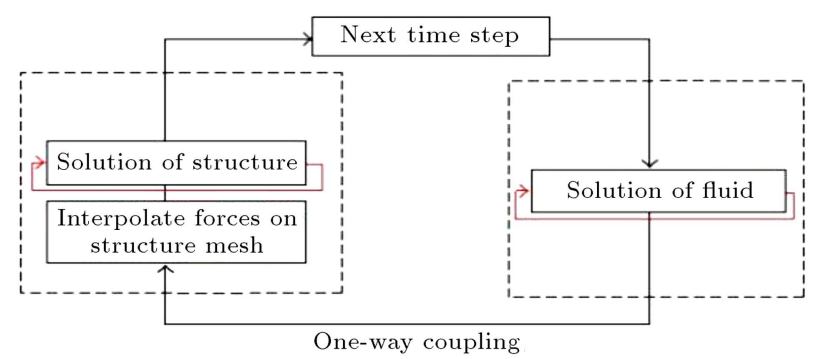

(a)

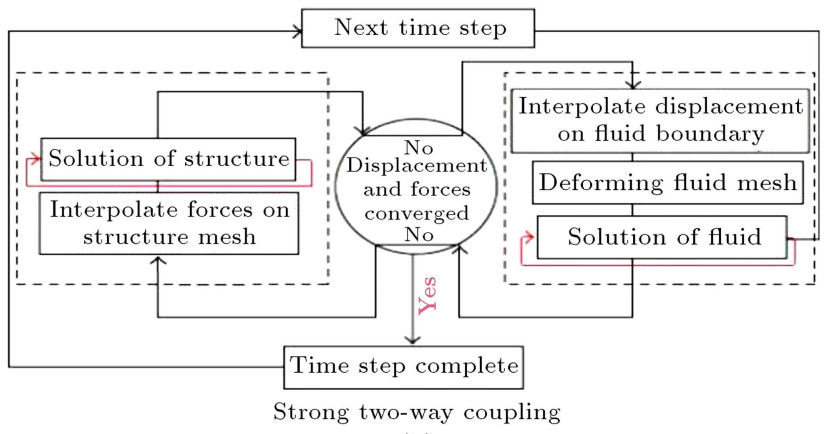

(b)

Figure 2. Solution algorithm for one-way and strong two-way coupling.

is a weak two-way coupling between the two fields and, even, a one-way coupling can be used.

Figure 3 shows what approach must be used in FSI issues. Given the above-mentioned issues, a twoway fully-coupled approach must be used to solve biomechanics issues [28].

\subsection{Formulation}

The governing equations for blood flow simulation are mass conservation equation (Eq. (1)) and momentum equation (Eq. (2)).

$$
\begin{aligned}
\nabla \cdot \vec{U} & =0 \\
\rho \frac{\partial u_{i}}{\partial t} & +\rho\left(\frac{\partial u_{i} \partial u_{j}}{\partial x_{j}}\right) \\
& =-\frac{\partial p}{\partial x_{i}}+\eta \frac{\partial}{\partial x_{j}}\left(\frac{\partial u_{i}}{\partial x_{j}}+\frac{\partial u_{j}}{\partial x_{i}}-\frac{2 \partial u_{k}}{3 \partial x_{k}} \delta_{i j}\right) .
\end{aligned}
$$

Laminar flow is considered fully developed in artery [29]. For this purpose, the Cartesian coordinates are 


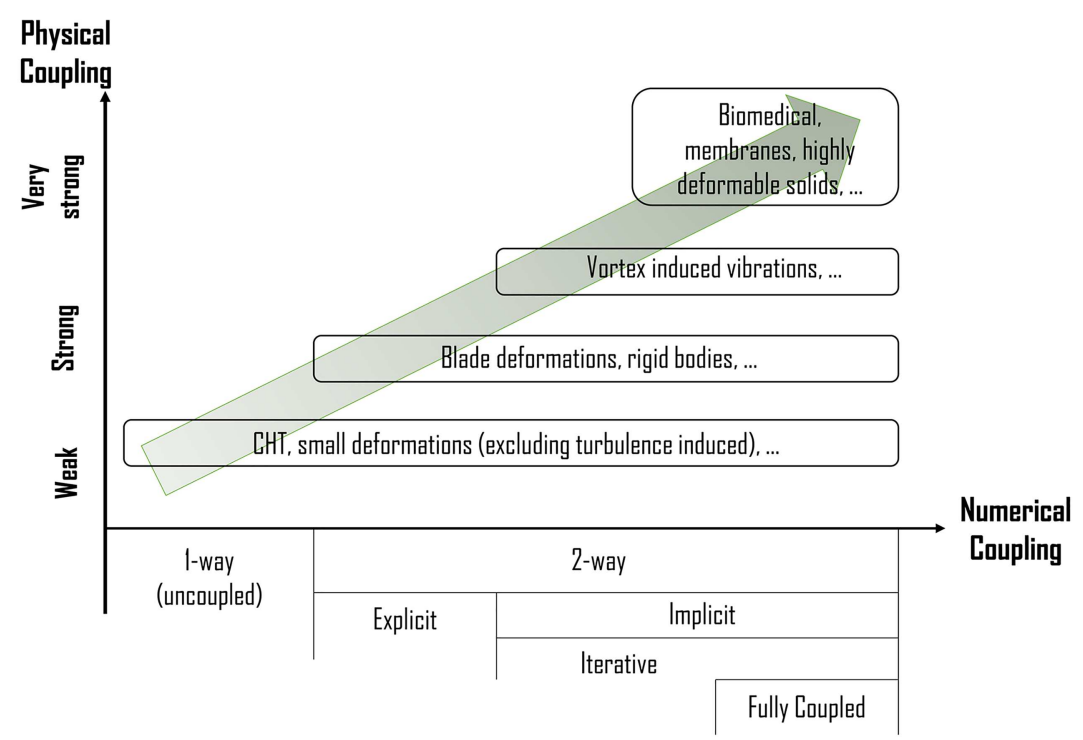

Figure 3. Division of Fluid-Solid Interaction (FSI) issues.

used. Since flow in the circular cross-section has axial symmetry, a differential ring as volume control is used.

\subsection{Boundary conditions}

The structure of the arterial wall is built in such a way that the elastic curve slope of the wall increases at high strains. This phenomenon gives nonlinear properties to arterial wall mechanic parameters, but the elastic behavior is suitable in the systolic-diastolic pressure range. Furthermore, the structural domain of an FSI problem typically exhibits nonlinear structural behavior. Such behavior can be classified as follows:

- Material nonlinearity: The stress-strain relationship is non-linear, e.g., for materials that exhibit plasticity;

- Geometric nonlinearity: The displacements and rotations are large and strains are either large or small.

In the current research, it is assumed that the structural material is homogeneous, isotropic, and linear elastic and is characterized by its Young's modulus, $E$, and Poisson ratio, $\nu$ (Eq. (3)). Elastic artery wall is assumed isotropic, which has been verified by researchers [30].

$$
D=\left[\begin{array}{ccc}
1 & \nu & 0 \\
\nu & 1 & 0 \\
0 & 0 & \frac{1-\nu}{2}
\end{array}\right] \frac{E}{1-\nu^{2}} .
$$

The boundary conditions for wall displacement areset such that in input and outlet flows, displacements are considered zero in the perpendicular direction. In the border areas of fluid, the solid-fluid interaction condition is used.

The boundary conditions of solid-fluid region state that solid and fluid displacements are equal.

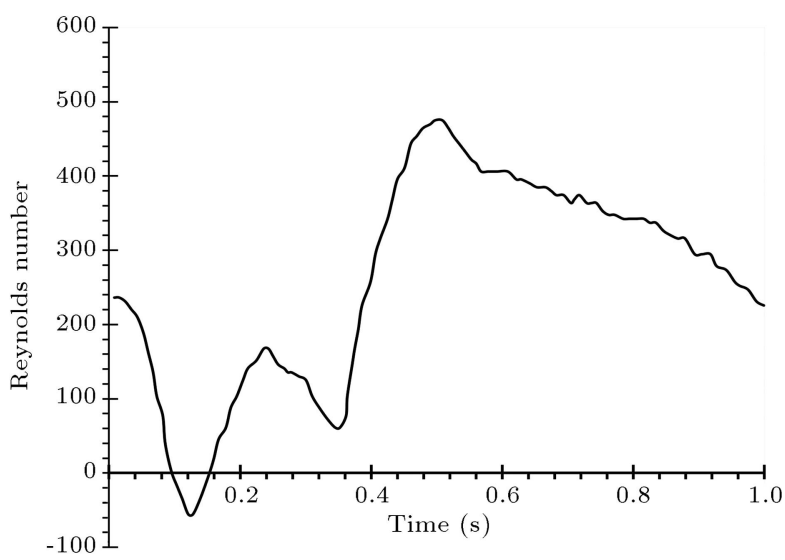

Figure 4. Flow Reynolds number according to time in unsteady flow.

Moreover, the fluid follows the non-slip condition. These conditions are shown in Eqs. (4) to (6) [31]:

$$
\begin{aligned}
& d_{s}=d_{f}, \\
& n \cdot \sigma_{s}=n \cdot \sigma_{f}, \\
& \dot{d}_{s}=\dot{d}_{f},
\end{aligned}
$$

where $d$ is displacement, $\sigma$ is the stress tensor, $n$ is perpendicular to the border, and subscripts $f$ and $s$ are related to the properties of the fluid and solid, respectively.

In the coronary input, the inlet velocity component along the $z$-axis is considered according to coronary pulse LAD [32], as depicted in Figure 3. In the steady state, the average velocity of pulsatile blood flow is considered as $0.217 \mathrm{~m} / \mathrm{s}$ according to average Reynolds number $=234.6$ [33]; moreover, the unsteady state is obtained, as given in Figure 4, and 
the average velocity of blood is obtained with respect to time. Finally, zero pressure gradients are used in the outlet.

\subsection{Numerical method}

The flow is considered three-dimensional and unsteady. The velocity pulse is used as the vessel input condition and artery wall elasticity. Moreover, blood is considered to be a Newtonian fluid. Blood density 1056 $\mathrm{kg} / \mathrm{m}^{3}$, dynamic viscosity $3.4186 \mathrm{CP}$, and linear elastic model are considered for the artery wall. To solve the mentioned equations, numerical methods of finite volume elements and ANSYS CFX software are used. The convergence criterion for the continuity equation is considered at $10^{-4}$. To eliminate the transition state, 10 cycles are used at the outset.

\subsection{Geometry}

Geometry of the problem consists of a straight pipe as the host vessel and a curve pipe as the connective vessel. Figure 5 shows the geometry and dimensions of the desired geometry with a bend angle.

In this study, three geometrical models are considered. The stenosis percentages of 30,50 , and 70 are taken into consideration. The stenosis length from the start to the end is considered $7 \mathrm{~mm}$ and the angle of $45^{\circ}$ is intended for the graft. The length of the entrance is chosen so that the fully developed blood flow reaches the stenosis. All geometrical properties in this study have been selected in line with available standards [34,35].

\subsection{Meshing and grid study}

To select a good meshing, the mesh convergence graph has been used. In this study, four different mesh configurations have been examined. The mesh convergence criterion is shear stress. The reason for choosing shear stress in the bed of the host vessel is its great significance and its role in stenosis. Chart data have been taken from the bed of the host vessel. The reason for choosing this area of the vessel is that it is just below the output bypass vessel, which is very important (see Figure 6).

In Figure 6, four different configurations are investigated. According to numbers 1 and 2, there is a very slight difference in shear stress. Grids smaller than meshing number 2 make almost no difference in the value of WSS and therefore, the meshing with 611156 elements (no. 2) is used in this study.

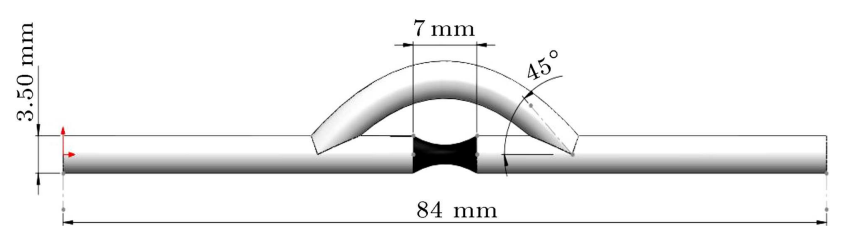

Figure 5. Complete coronary artery bypass graft geometry.

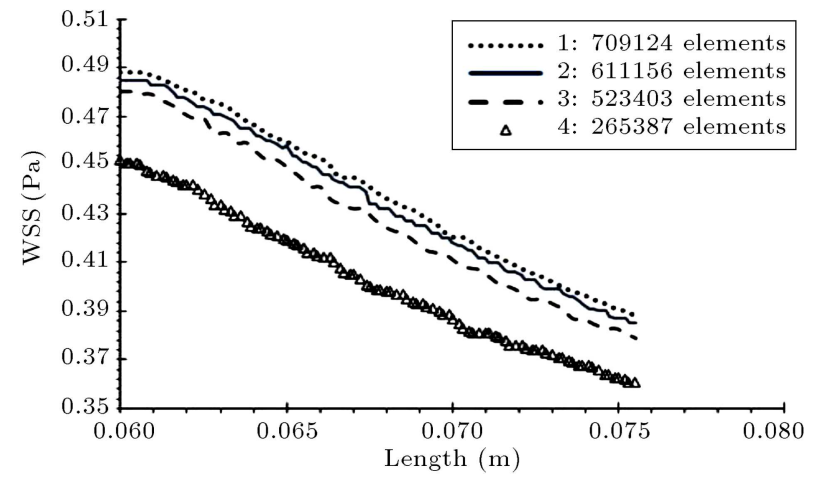

Figure 6. Wall Shear Stress (WSS) along the artery wall for different grid configurations.

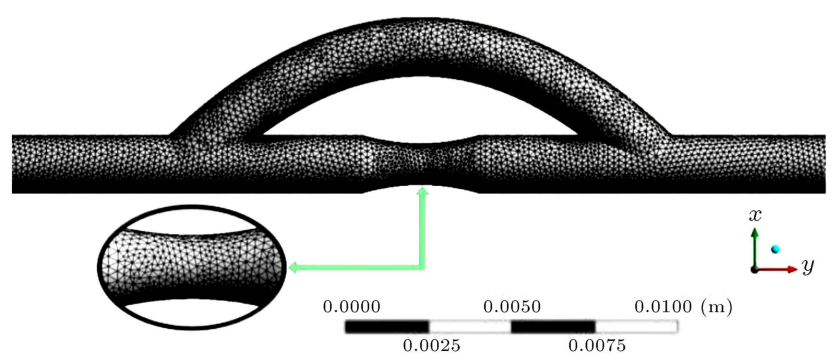

Figure 7. Computational grid using inflation.

In this type of meshing for geometry, the orthogonal parameter is 0.87 . This parameter shows the optimization of the placement of elements next to each other. The value of this parameter ranges between 0 and 1 and if this value is larger, the reticulation geometry will be better. To ensure a better analysis of the areas of the boundary layer, the grid with a dimension of 8 microns that can cover these areas is used, and for the grid setting of the boundary layer area, the inflation grid is used to obtain more accurate results. The computational grid is shown in Figure 7.

\subsection{Validation of results}

Figure 8 shows the WSS along the artery length. In this figure, the result obtained by Ko et al. [33] is also shown. As shown earlier, very good agreement is obtained.

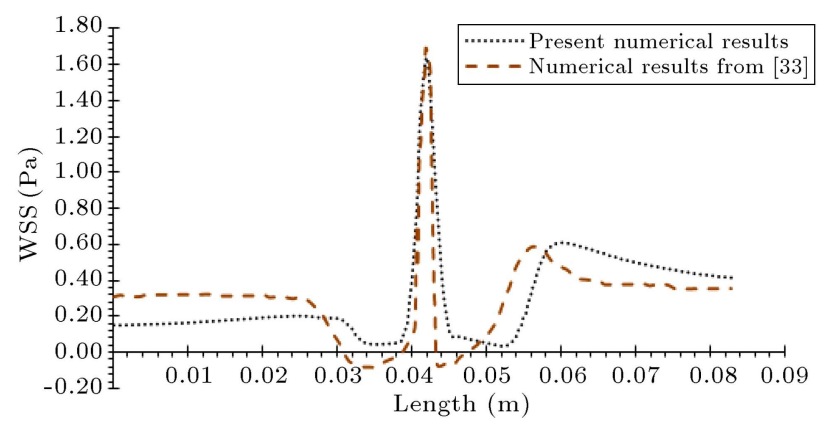

Figure 8. Examining shear stress in the bed of coronary artery: Two rigid bodies with stenosis $70 \%$. 


\section{Results and discussion}

In this section, the effects of the elasticity of the artery wall and unsteady flow case on WSS, streamline, and blood velocity in different stenosis percentages are examined.

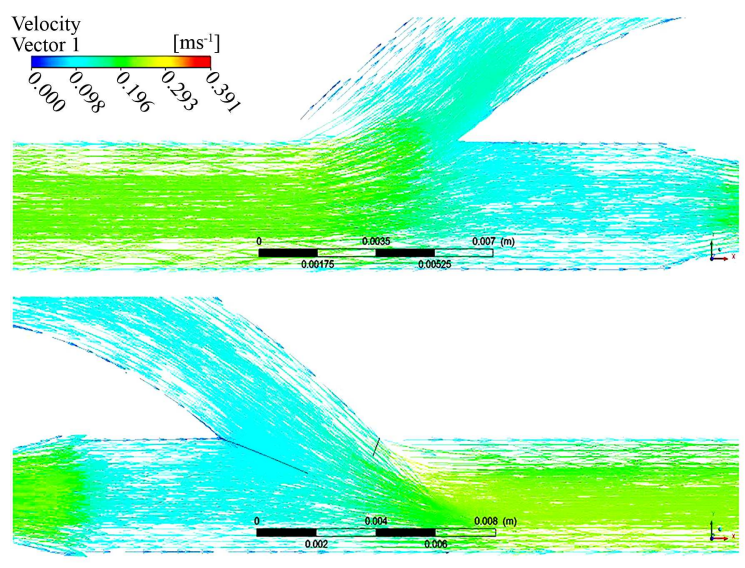

(a)

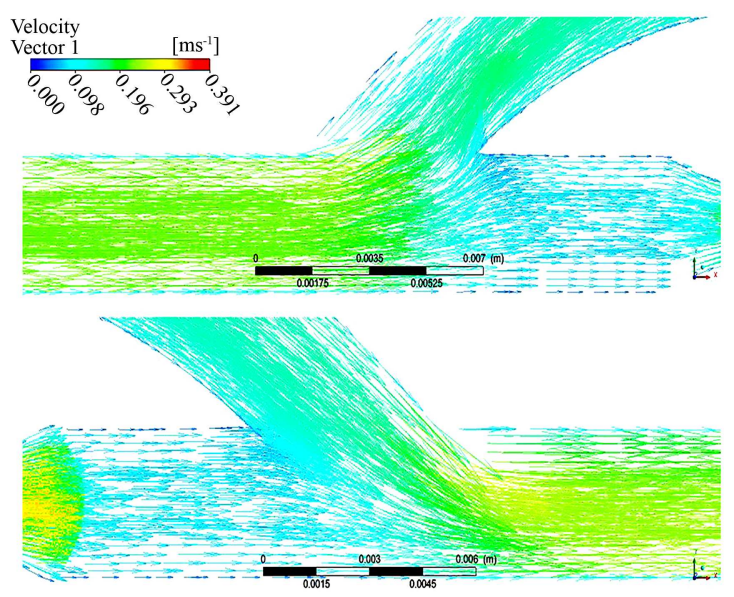

(c)

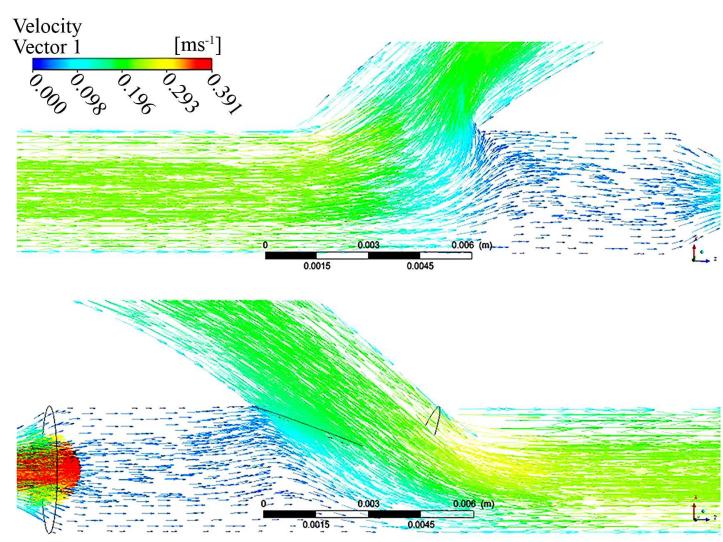

(e)

\subsection{Steady state case}

Figure 9 shows the streamlines in distal and proximal areas for the three geometric models $(30 \%, 50 \%$, and $70 \%$ ) for two cases: rigid and elastic artery walls. The reason for investigating the streamlines is the importance of rotational flow. It is noteworthy that
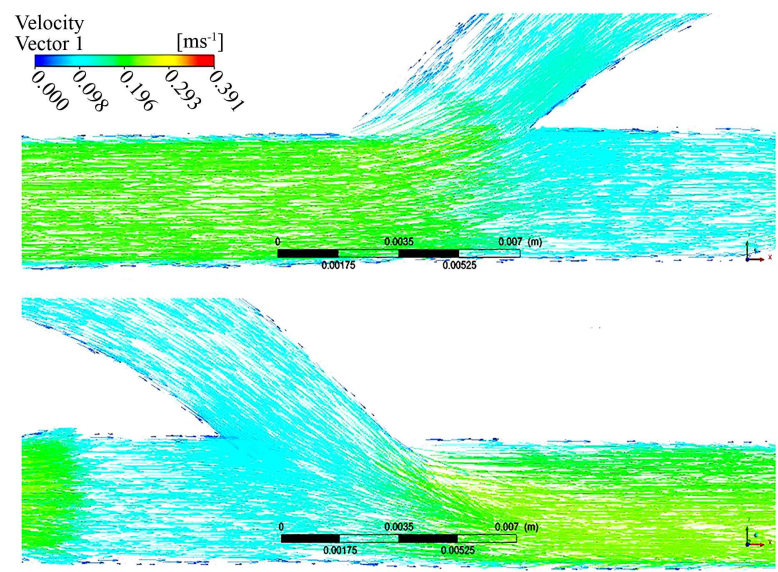

(b)
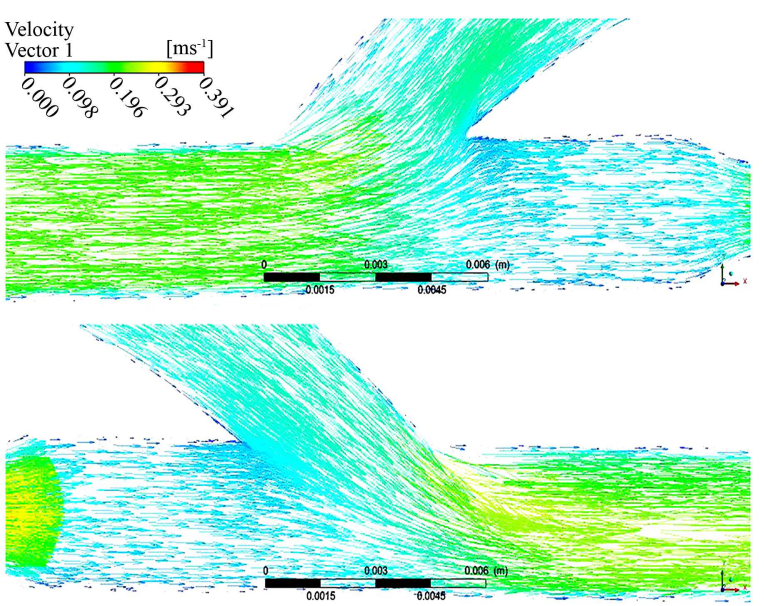

(d)

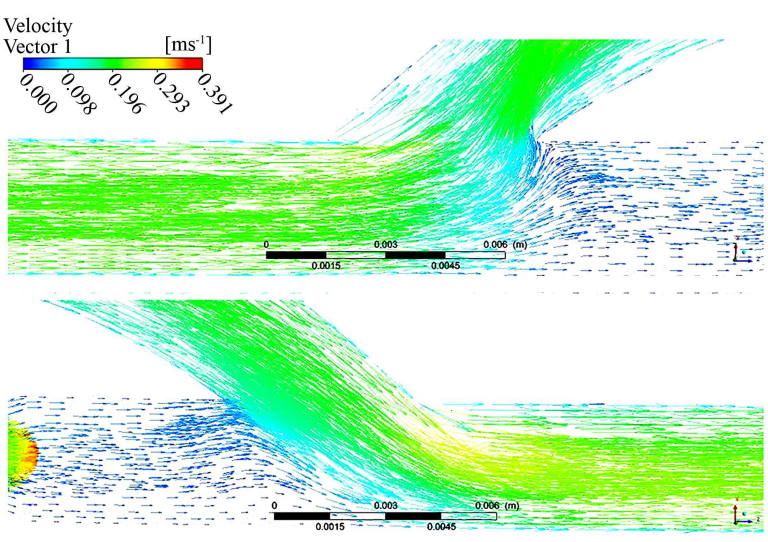

(f)

Figure 9. Streamlines in the distal and proximal areas: (a) $30 \%$ stenosis-rigid wall, (b) $30 \%$ stenosis-elastic wall, (c) $50 \%$ stenosis-rigid wall, (d) $50 \%$ stenosis-elastic wall, (e) $70 \%$ stenosis-rigid wall, and (f) $70 \%$ stenosis-elastic wall. 


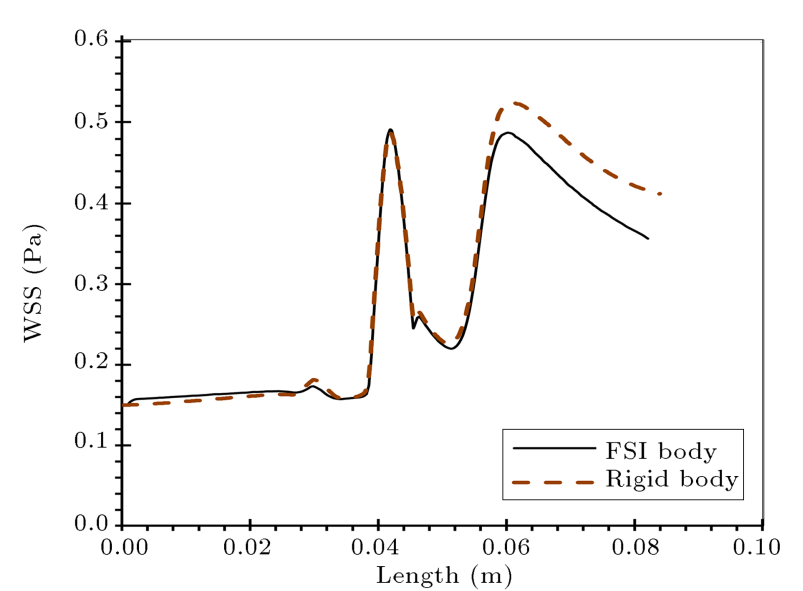

Figure 10. Wall Shear Stress (WSS) at the bed of the host artery for rigid and elastic walls with $30 \%$ stenosis.

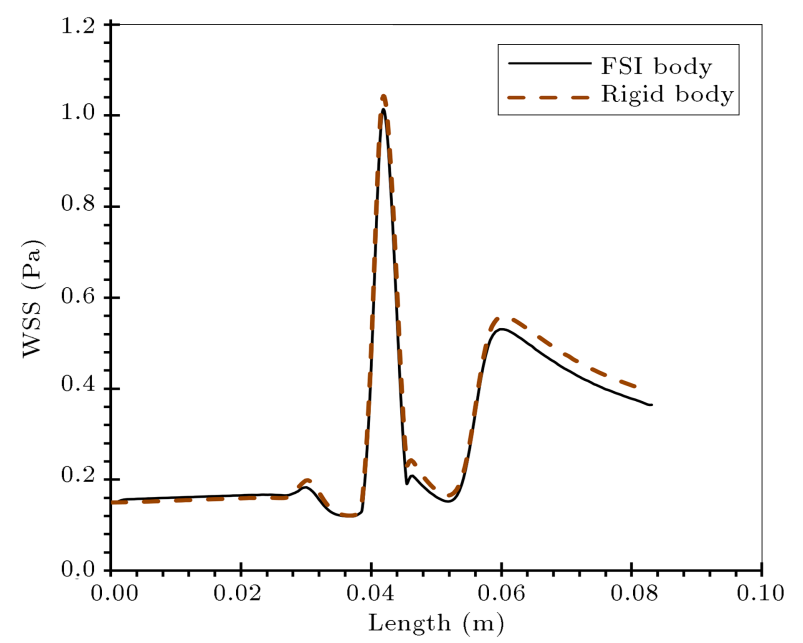

Figure 11. Wall Shear Stress (WSS) at the bed of the host artery for rigid and elastic walls with $50 \%$ stenosis.

rotational flow causes endothelial cells to accumulate and ultimately, leads to stenosis. It is observed that streamlines under the toe of distal and proximal areas are non-uniform.

Figure 10 shows WSS in the bed of the host vessel for two cases, rigid and elastic artery walls, with $30 \%$ stenosis. The amounts of shear stress in the bed of the host vessel just below the distal anastomosis region are $0.49 \mathrm{~Pa}$ and $0.591 \mathrm{~Pa}$ for the elastic artery wall and the vessel with a rigid wall, respectively. Therefore, WSS is $17 \%$ lower when the FSI, is considered, whereas the shear stress in the bed of the host vessel between rigid and elastic cases in the presence of $50 \%$ stenosis has just $2 \%$ differences. Such a stress occurs following the distal anastomosis (see Figure 11). Based on a comparison of shear stress in the bed of the host vessel between rigid and elastic cases with $70 \%$ stenosis, Figure 12, maximum shear stresses in the elastic and rigid artery walls are $1.582 \mathrm{~Pa}$ and $1.807 \mathrm{~Pa}$. In other words, the wall stress in this region was reduced by $12.5 \%$.

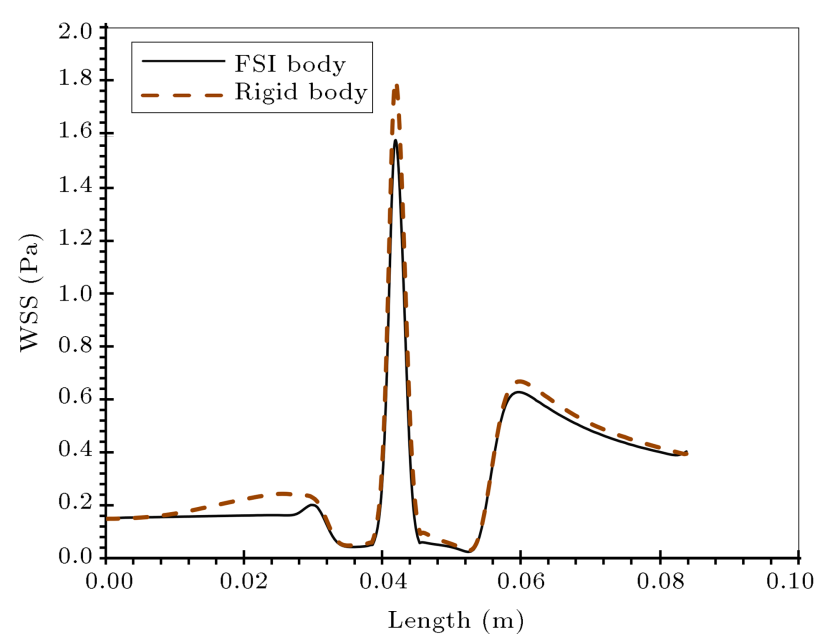

Figure 12. Wall Shear Stress (WSS) at the bed of artery for the rigid and elastic walls with $70 \%$ stenosis.

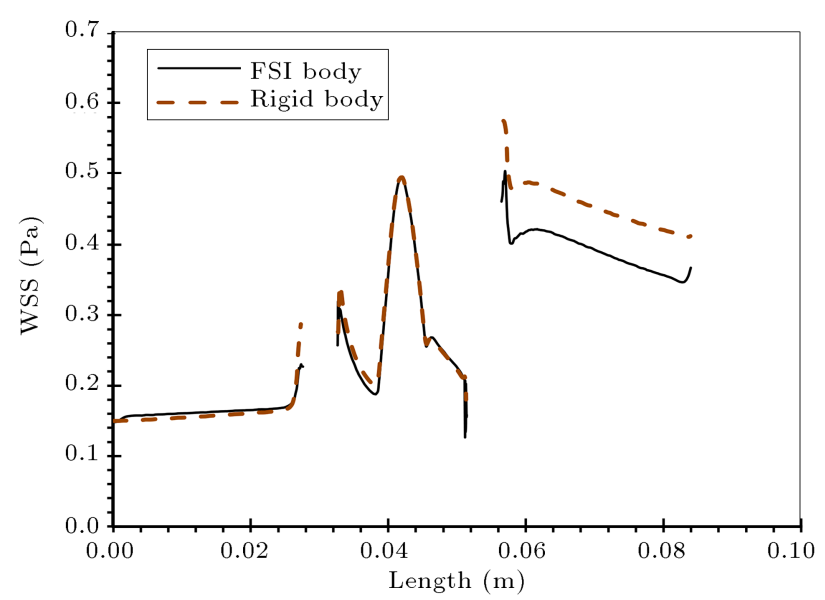

Figure 13. Wall Shear Stress (WSS) at the toe and heel for rigid and elastic cases with $30 \%$ stenosis.

Moreover, before proximal bifurcation, the shear stress of rigid artery wall was increased by $40 \%$.

By comparing the WSS at toe and heel in the presence of $30 \%$ stenosis as shown in Figure 13, it is observed that for the rigid artery wall compared to the elastic artery wall at the toe of distal anastomosis, WSS increased by $19 \%$. Moreover, as depicted in Figure 14, in the presence of $50 \%$ stenosis, it can be concluded that at the toe of the distal area, WSS was reduced by $16 \%$ for the elastic artery wall compared to the rigid artery wall. Moreover, by comparing the WSS at the toe and heel areas between rigid and elastic artery walls with the presence of $70 \%$ stenosis, the elastic WSS was reduced by $12 \%$ compared to the rigid artery wall in the stenosis region. Moreover, elastic WSS at the toe of proximal bifurcation was $32 \%$ lower than that at the rigid artery wall (see Figure 15).

Figure 16 shows the WSS in the bed of the vessel for different percentages of stenosis for the rigid artery wall. It can be concluded that the WSS 


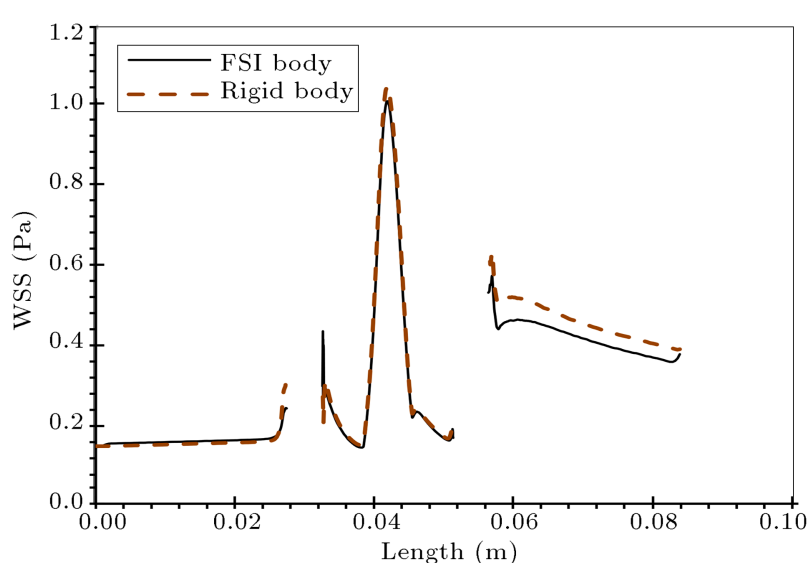

Figure 14. Wall Shear Stress (WSS) at the toe and heel for rigid and elastic cases with $50 \%$ stenosis.

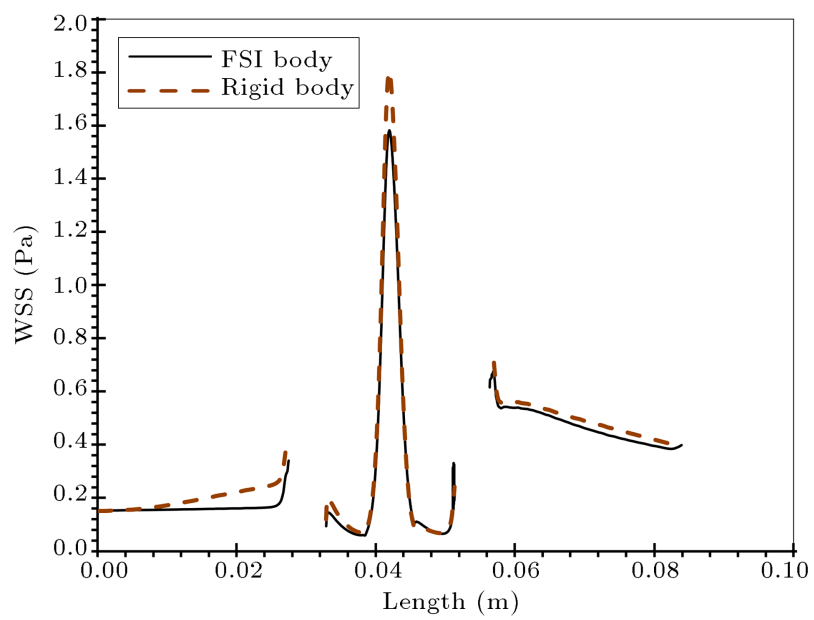

Figure 15. Wall Shear Stress (WSS) at the toe and heel for rigid and elastic cases with $70 \%$ stenosis.

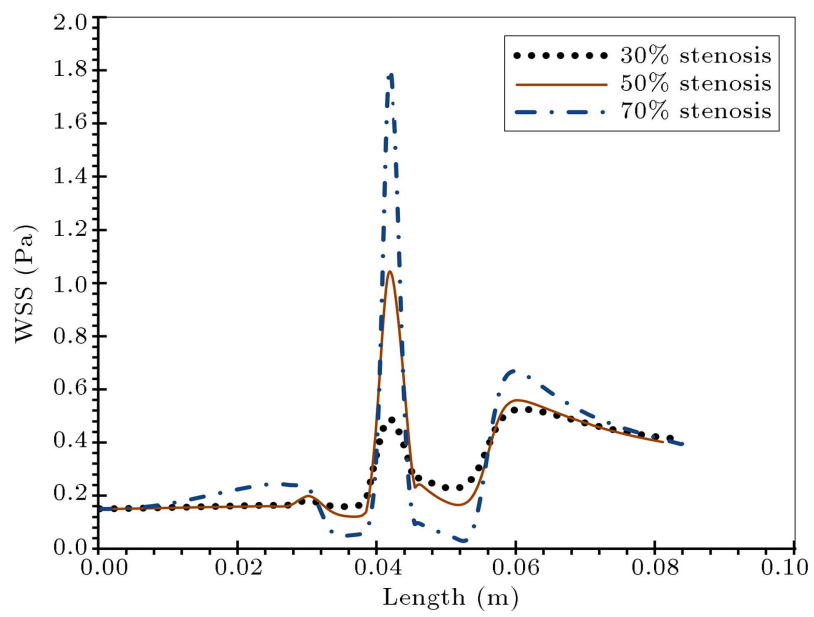

Figure 16. Wall Shear Stress (WSS) at the bed of the host vessel for three stenoses with the rigid wall.

for $50 \%$ stenosis is $55 \%$ compared to that for $70 \%$ stenosis. It was shown that with a $20 \%$ increase in the stenosis, an $80 \%$ increase in the maximum WSS was observed. Further, for a comparison of $50 \%$ and

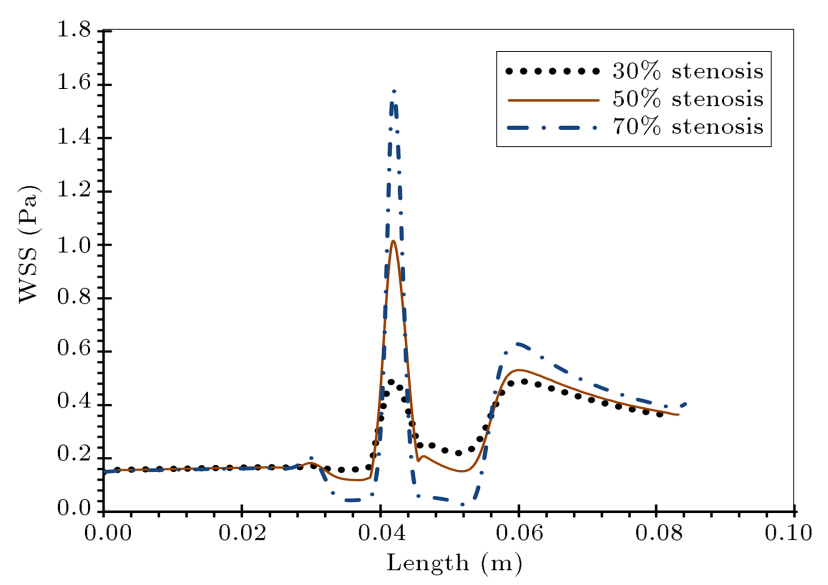

Figure 17. Wall Shear Stress (WSS) at the bed of the host vessel for three stenoses with the elastic wall.

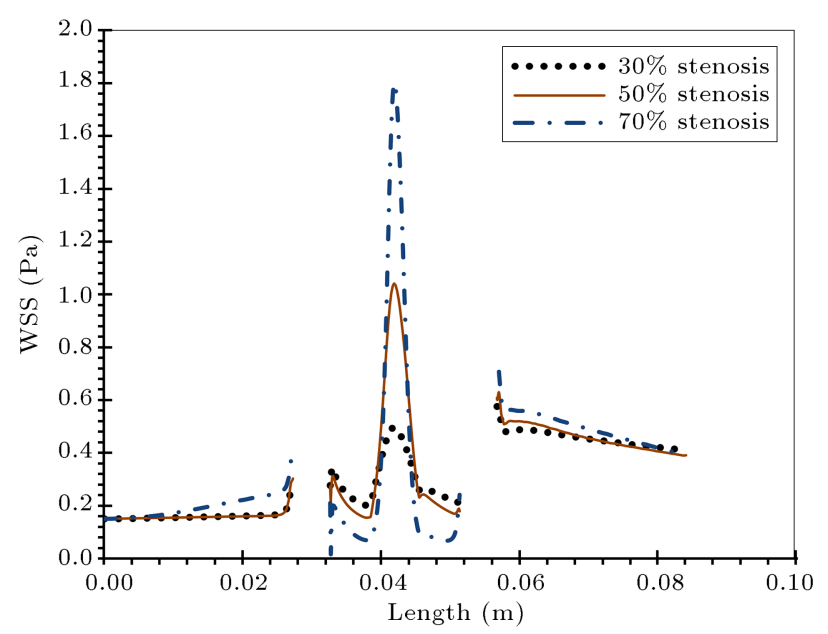

Figure 18. Wall Shear Stress (WSS) at the toe and heel of the host vessel for three stenoses with the rigid wall.

$30 \%$ rates of stenosis, it was concluded that WSS for $50 \%$ stenosis was almost twice as much as that for $30 \%$. Minimum WSS between these three cases is $0.03 \mathrm{~Pa}$, which occurs in the vessel bed right below the distal anastomosis for the $70 \%$ stenosis. In Figure 17 , there is a significant difference in WSS in the stenosis region, which indicates that if the stenosis percentage increases, the minimum and maximum of WSS occur at the maximum stenosis percentage.

By comparing WSS with different stenosis percentages in toe and heel regions with a rigid artery wall, it can be concluded that as stated in the previous sections, minimum WSS may occur in case of $70 \%$ stenosis with its value being $16 \%$ that of $30 \%$. In regions between stenosis and distal heel, the WSS decreases drastically. In the heel of distal anastomosis, the value of WSS for $30 \%$ stenosis is four times more than that of $70 \%$ stenosis; however, this difference is not very significant when $30 \%$ and $50 \%$ rates of stenosis are compared. Moreover, the shear stress for $30 \%$ stenosis has $10 \%$ less than that for $50 \%$ (see Figure 18). 


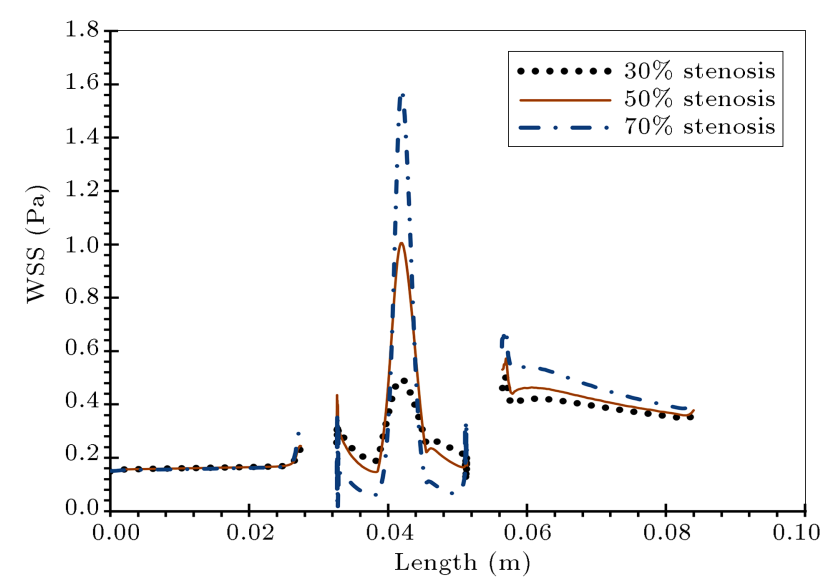

Figure 19. Wall Shear Stress (WSS) at the toe and heel of the host vessel for three stenoses with the elastic wall.

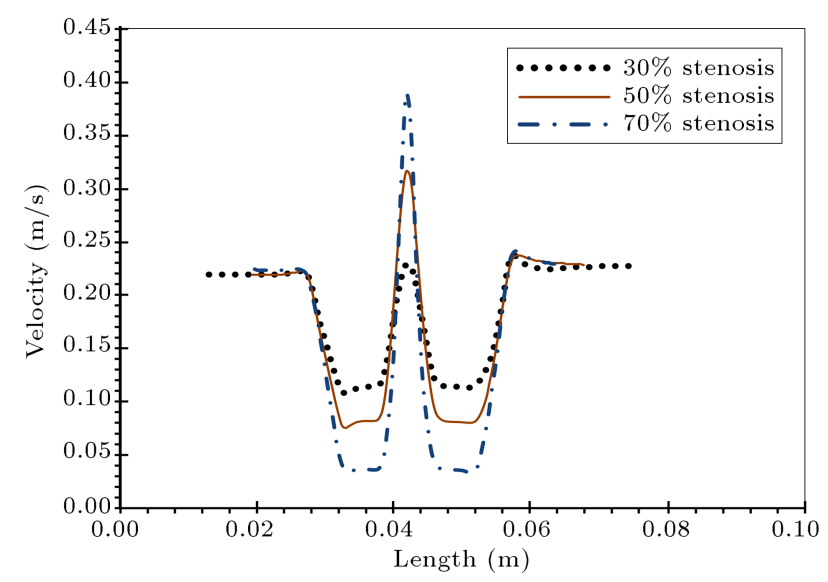

Figure 20. Blood velocity for different stenoses for the rigid wall.

Figure 19 shows the WSS for different stenosis percentages in the toe and heel regions for elastic and solid artery walls. In the proximal heel, the WSS for $30 \%$ stenosis is four times more than that for $70 \%$. However, the differences are not significant between $30 \%$ and $50 \%$ cases.

In Figure 20, based on a comparison of the blood velocity for different percentages of stenosis at the rigid artery wall, one can conclude that velocity in the region between distal anastomosis and 50\% stenosis is twice more than that for $70 \%$ stenosis. For $30 \%$ stenosis, this value increases three times. In the stenosis region where the maximum velocity occurs, the velocity increases by $22 \%$ for $70 \%$ stenosis compared to that for $50 \%$ and increases by $72 \%$ compared to that for $30 \%$ stenosis. By comparing blood velocity at different percentages of stenosis $(30 \%, 50 \%$, and $70 \%$ stenosis ) at the elastic artery wall, it can be concluded that in the stenosis region for $70 \%$ stenosis, the velocity increases by $10 \%$ compared to that for $50 \%$. This amount is $10 \%$ less than that at the rigid artery wall.

After comparing blood velocity at the rigid and elastic artery walls with $30 \%$ stenosis, it is concluded

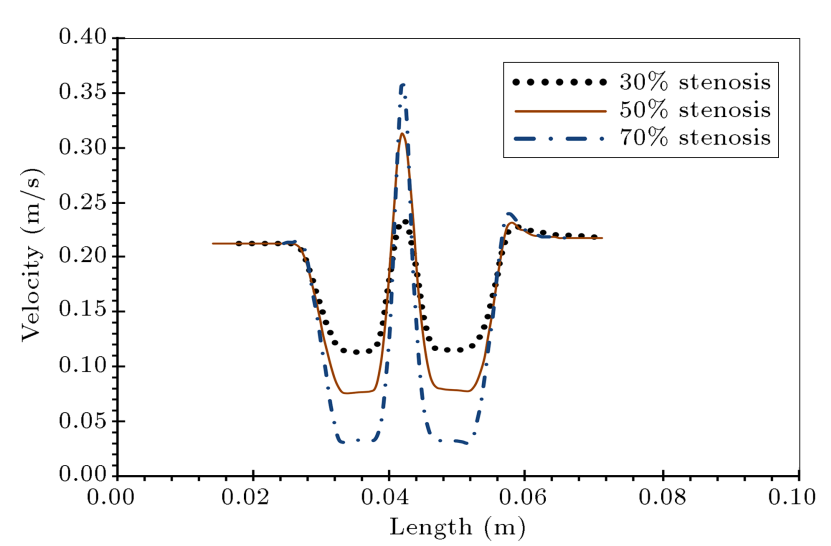

Figure 21. Blood velocity for different stenoses for the elastic wall.

that in the toe region of proximal bifurcation and distal anastomosis, the velocity is $10 \%$ higher at the rigid artery wall than that at the elastic artery wall. Based on a comparison of the velocity at the rigid and elastic artery walls for $70 \%$, it can be observed that for the rigid artery wall at the toe of proximal bifurcation, the velocity is $10 \%$ greater than that at the elastic artery wall. This difference reaches $12 \%$ in the stenosis region (see Figure 21).

\subsection{Unsteady case}

The results for the unsteady case for $70 \%$ stenosis are given here when the input blood velocity is pulsatile, as stated earlier. The flow does not follow a uniform pattern in the distal and proximal areas. It is shown that rotational flow occurs under the heel of distal anastomosis. Because of considering the pulsatile blood flow, the results are closer to reality than that of the steady case. The results are shown in different time zones (see Figure 22).

As shown in Figure 23, by comparing the blood velocity at the host vessel in the steady and unsteady cases, one can observe that in the proximal and distal toe regions, the velocity is higher than $10 \%$ in the steady state case. The highest difference can be seen in the stenosis region in which the velocity for steady flow has increased by $34 \%$.

Figure 24 shows the vessel bed WSS in the unsteady and steady cases. As shown earlier, the WSS in the unsteady case in the stenosis region is $41.3 \%$ higher than that in the steady case, while WSS percentages are about the same in other regions. Similarly, as shown in Figure 25, for the host vessel, the differences between the unsteady and steady solutions are calculated at $41.3 \%$ in the middle of the host vessel.

\section{Conclusion}

The obtained results of this study indicated that the application of Fluid-Solid Interaction (FSI) condition considering pulsatility of flow had significant effect on 

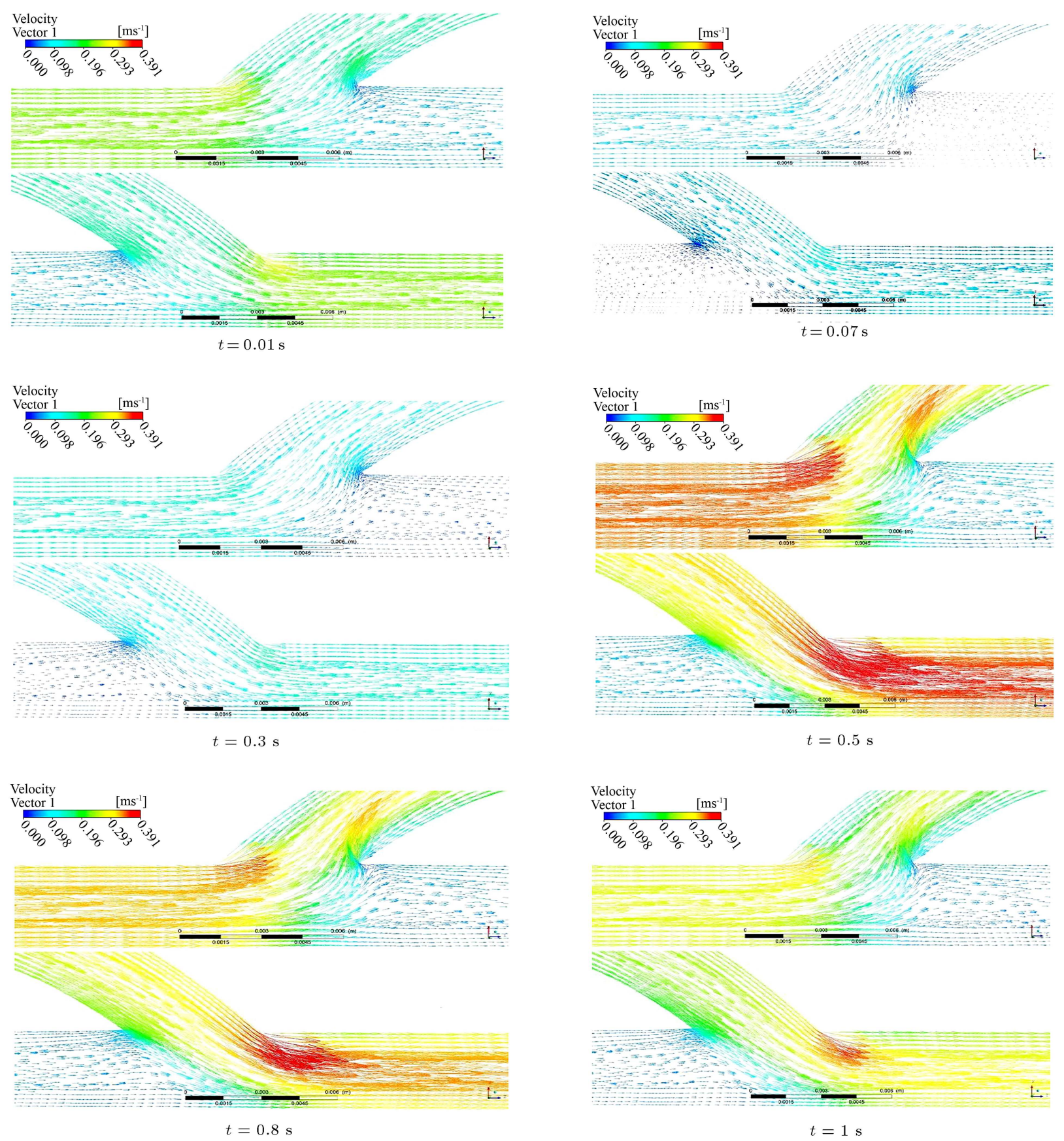

Figure 22. Streamlines in different time zones.

flow hemodynamics. Moreover, using velocity pulse for flow demonstrated fluctuations and using steady flow assumption caused errors in the stenosis region.

By examining the Wall Shear Stress (WSS) values of the vessel bed in $30 \%$ and $50 \%$ stenosis cases, there was not a significant difference between the rigid and elastic cases; however, the difference was more pronounced for $70 \%$ stenosis in the proximal bifurcation and stenosis regions. Furthermore, much blood flow entered the graft from the proximal region in higher percentages of stenosis and the streamlines of the flow became non-uniform and affected WSS values. In FSI consideration, as mentioned before, changes are more obvious. It can be concluded that upon decreasing the stenosis percentage, the difference of WSS values at the distal toe between the rigid and elastic cases became more obvious.

For the blood velocity values at the host vessel, there is no significant difference between rigid and elastic cases in the steady state flow. Therefore, the rigid artery wall assumption is acceptable for the steady state condition to investigate velocity magnitude.

By considering velocity pulse as an input boundary condition (unsteady state), there are significant 


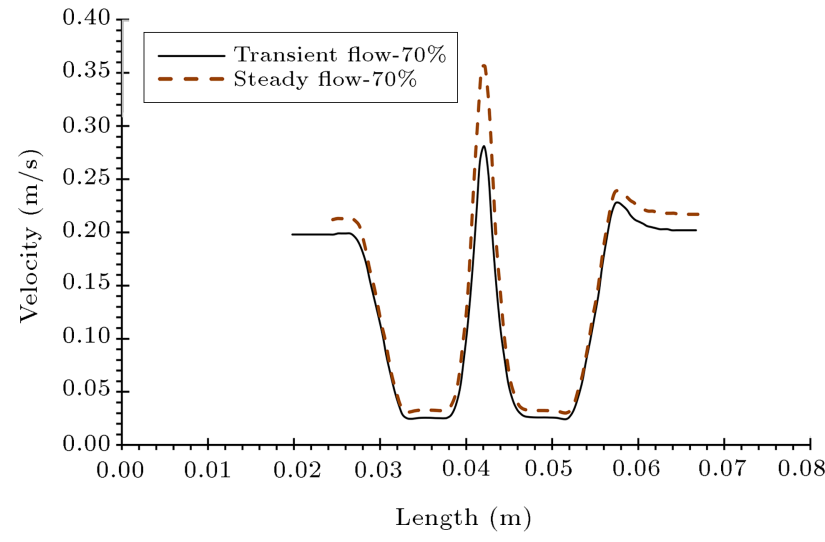

Figure 23. Blood velocity in the host vessel with $70 \%$ stenosis for the unsteady state case.

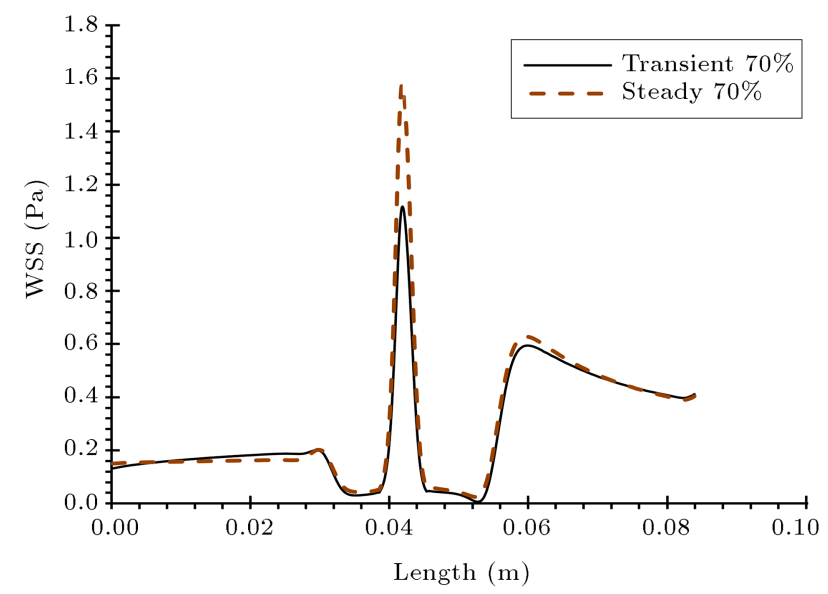

Figure 24. Wall Shear Stress (WSS) for unsteady and steady cases on host vessel bed.

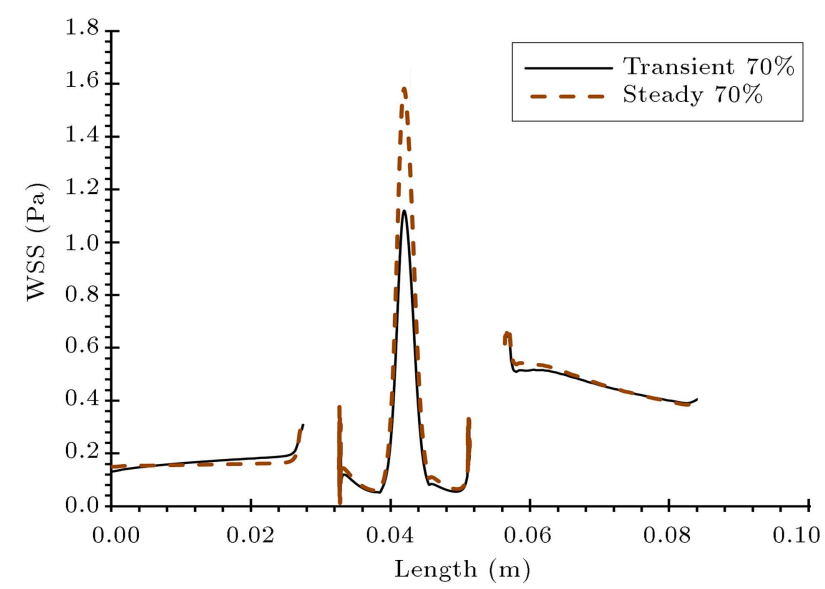

Figure 25. Wall Shear Stress (WSS) for unsteady and steady cases at the host vessel upper wall.

differences between the WSS values and velocity magnitudes in the stenosis region as a result of pulsatility. Therefore, the most effective part of velocity pulse is in the stenosis region. It is not very influential in analyzing proximal and distal areas and one can use steady flow for considering these regions.

$\begin{array}{ll}\text { Nomenclature } \\ d_{s} & \text { Solid displacement } \\ d_{f} & \text { Fluid displacement } \\ D & \text { Deformation rate tensor } \\ \text { FSI } & \text { Fluid-Solid Interaction } \\ P & \text { Pressure } \\ \vec{U} & \text { Velocity vector } \\ \sigma_{s} & \text { Solid shear stress } \\ \sigma_{f} & \text { Fluid shear stress } \\ \rho & \text { Density } \\ \eta & \text { Dynamic viscosity } \\ E & \text { Young's modulus } \\ \nu & \text { Poisson ratio }\end{array}$

\section{Acknowledgment}

The authors would like to thank the Department of Mechanical Engineering at Foulad Institute of Technology of Iran for its support.

\section{References}

1. Kaplan, H., Thomson, R.C., Trumble, B.C., et al. "Coronary atherosclerosis in indigenous South American Tsimane: A cross-sectional cohort study", The Lancet, 389, pp. 1730-1739 (2017).

2. Weydahl, E.S. and Moore Jr, J.E. "Dynamic curvature strongly affects wall shear rates in a coronary artery bifurcation model", Journal of Biomechanics, 34, pp. 1189-1196 (2001).

3. Ku, D.N. "Blood flow in arteries", Annual Review of Fluid Mechanics, 29, pp. 399-434 (1997).

4. Kraiss, L.W., Kirkman, T.R., Kohler, T.R., Zierler, B., and Clowes, A.W. "Shear stress regulates smooth muscle proliferation and neointimal thickening in porous polytetrafluoroethylene grafts", Arteriosclerosis and Thrombosis: A Journal of Vascular Biology, 11, pp. 1844-1852 (1991).

5. Ku, D.N., Giddens, D.P., Zarins, C.K., and Glagov, $\mathrm{S}$. "Pulsatile flow and atherosclerosis in the human carotid bifurcation. Positive correlation between plaque location and low oscillating shear stress", Arteriosclerosis: An Official Journal of the American Heart Association, Inc. 5, pp. 293-302 (1985).

6. Jin, C. and Liu, Y. "Influence of competitive flow caused by different stenosis on coronary artery bypass hemodynamics and PIV study", Molecular and Cellular Biomechanics, 16, pp. 51-52 (2019).

7. Duncker, D.J., Koller, A., Merkus, D., and Canty Jr, J.M. "Regulation of coronary blood flow in health and ischemic heart disease", Progress in Cardiovascular Diseases, 57, pp. 409-422 (2015). 
8. Bertolotti, C., Deplano, V.r., Fuseri, J., and Dupouy, P. "Numerical and experimental models of postoperative realistic flows in stenosed coronary bypasses", Journal of Biomechanics, 34, pp. 1049-1064 (2001).

9. Bonert, M., Myers, J.G., Fremes, S., Williams, J., and Ethier, C.R. "A numerical study of blood flow in coronary artery bypass graft side-to-side anastomoses", Annals of Biomedical Engineering, 30, pp. 599-611 (2002).

10. Ethier, C.R., Steinman, D., Zhang, X., Karpik, S. and Ojha, M. "Flow waveform effects on end-to-side anastomotic flow patterns", Journal of Biomechanics, 31, pp. 609-617 (1998).

11. Song, M.-H., Sato, M., and Ueda, Y. "Threedimensional simulation of coronary artery bypass grafting with the use of computational fluid dynamics", Surgery Today, 30, pp. 993-998 (2000).

12. Zohravi, E., Shirani, E., and Sadeghi, M. "Hemodynamic analysis of pulsatile blood flow in a complete bypass graft with different anastomosis angles", Scientia Iranica, Transaction B, Mechanical Engineering, 22, p. 423 (2015).

13. Fung, Y.-C. "Biomechanics: circulation", Shock, 9, p. 155 (1998).

14. Cebral, J.R., Yim, P.J., Löhner, R., Soto, O., and Choyke, P.L. "Blood flow modeling in carotid arteries with computational fluid dynamics and MR imaging", Academic Radiology, 9, pp. 1286-1299 (2002).

15. De Hart, J., Peters, G., Schreurs, P., and Baaijens, F. "A three-dimensional computational analysis of fluid structure interaction in the aortic valve", Journal of Biomechanics, 36, pp. 103-112 (2003).

16. Liu, G., Wu, J., Ghista, D.N., Huang, W., and Wong, K.K. "Hemodynamic characterization of transient blood flow in right coronary arteries with varying curvature and side-branch bifurcation angles", Computers in Biology and Medicine, 64, pp. 117-126 (2015).

17. Doost, S.N., Ghista, D., Su, B., Zhong, L., and Morsi, Y.S. "Heart blood flow simulation: a perspective review", Biomedical Engineering Online, 15, p. 101 (2016).

18. He, X. and Ku, D.N. "Pulsatile flow in the human left coronary artery bifurcation: average conditions", Journal of Biomechanical Engineering, 118, pp. 74-82 (1996).

19. Rindt, C. and Steenhoven, A. "Unsteady flow in a rigid 3-D model of the carotid artery bifurcation", Journal of Biomechanical Engineering, 118, pp. 90-96 (1996).

20. Weston, S., Wood, N., Tabor, G., Gosman, A., and Firmin, D. "Combined MRI and CFD analysis of fully developed steady and pulsatile laminar flow through a bend", Journal of Magnetic Resonance Imaging, 8, pp. 1158-1171 (1998).

21. Loudon, C. and Tordesillas, A. "The use of the dimensionless Womersley number to characterize the unsteady nature of internal flow", Journal of Theoretical Biology, 191, pp. 63-78 (1998).

22. Wentzel, J.J., Whelan, D.M., van der Giessen, W.J., et al. "Coronary stent implantation changes 3-D vessel geometry and 3-D shear stress distribution", Journal of Biomechanics, 33, pp. 1287-1295 (2000).

23. Leuprecht, A., Perktold, K., Prosi, M., et al. "Numerical study of hemodynamics and wall mechanics in distal end-to-side anastomoses of bypass grafts", Journal of Biomechanics, 35, pp. 225-236 (2002).

24. Beier, S., Ormitson, J., Webster, M., et al. "Impact of bifurcation angle and other anatomical characteristics on blood flow-A computational study of non-stented and stented coronary arteries", Journal of Biomechanics, 49, pp. 1570-1582 (2016).

25. Ballarin, F., Faggiano, E., Manzoni, A., et al. "Numerical modeling of hemodynamics scenarios of patientspecific coronary artery bypass grafts", Biomechanics and Modeling in Mechanobiology, 16, pp. 1373-1399 (2017).

26. Guerciotti, B., Vergara, C., Ippolito, S., et al. "A computational fluid-structure interaction analysis of coronary Y-grafts", Medical Engineering \& Physics, 47, pp. 117-127 (2017).

27. Benra, F.-K., Dohmen, H.J., Pei, J., Schuster, S., and Wan, B. "A comparison of one-way and two-way coupling methods for numerical analysis of fluid-structure interactions", Journal of Applied Mathematics, 2011, pp. 1-16 (2011).

28. Bolukbasi, A., Athari, H., and Ciloglu, D. "The application of FSI Techniques in modeling of realist pulmonary systems", World Academy of Science, Engineering and Technology, International Journal of Mechanical, Aerospace, Industrial, Mechatronic and Manufacturing Engineering, 9, pp. 1064-1069 (2015).

29. Jahangiri, M., Saghafian, M., and Sadeghi, M.R. "Numerical study of turbulent pulsatile blood flow through stenosed artery using fluid-solid interaction", Computational and Mathematical Methods in Medicine, 2015, pp. 1-10 (2015).

30. Shaik, E., Numerical Simulations of Blood Flow in Arteries Using Fluid-Structure Interactions, Wichita State University (2007).

31. Savabi, R., Nabaei, M., Farajollahi, S., and Fatouraee, N. "Fluid structure interaction modeling of aortic arch and carotid bifurcation as the location of baroreceptors", International Journal of Mechanical Sciences, 165, p. 105222 (2020).

32. Steinman, D., Vinh, B., Ethier, C.R., et al. "A numerical simulation of flow in a two-dimensional endto-side anastomosis model", Journal of Biomechanical Engineering, 115, pp. 112-118 (1993). 
33. Ko, T., Ting, K., and Yeh, H. "Numerical investigation on flow fields in partially stenosed artery with complete bypass graft: An in vitro study", International Communications in Heat and Mass Transfer, 34, pp. 713-727 (2007).

34. Giannoglou, G., Soulis, J., Farmakis, T., Farmakis, D., and Louridas, G. "Haemodynamic factors and the important role of local low static pressure in coronary wall thickening", International Journal of Cardiology, 86, pp. 27-40 (2002).

35. Leung, W.-H., Stadius, M.L., and Alderman, E.L. "Determinants of normal coronary artery dimensions in humans", Circulation, 84, pp. 2294-2306 (1991).

\section{Biographies}

Hooman Fallahi received his BS degree from Islamic Azad University of Marvdasht, Iran in 2013 and his MS degree from Foulad Institute of Technology, Iran in 2016, all in Mechanical Engineering. His research interests include biomechanics, cardiovascular modeling, turbulence and turbulence modeling, and multiphase flow.
Ebrahim Shirani is a Professor of Mechanical Engineering at Isfahan University of Technology, Iran. He received his BS degree from Sharif University of Technology, Iran in 1975 and his MS and PhD degrees from Stanford University, USA in 1977 and 1981, respectively, all in Mechanical Engineering. His research interests include computational fluid dynamics, computational micro and nano-fluid dynamics, turbulence and turbulence modeling and numerical simulation of interfacial flows, turbo machinery, and biofluid dynamics. He is the author of 12 books and has published over 50 journal and several conference papers. He is also currently a faculty member at Foulad Institute of Technology, Fouladshahr, Isfahan, Iran.

Elnaz Zohravi is a Researcher at Isfahan University of Technology. She received her BS, MS, and PhD degrees from the Mechanical Engineering Department at Isfahan University of Technology, Iran in 2006, 2009, and 2018, respectively. Her research interests include biomechanics, cardiovascular modeling, and nano-fluid dynamics. 